\title{
ON STANDARD SUBGROUPS OF TYPE ${ }^{2} E_{6}(2)$
}

\author{
G. STROTH
}

\begin{abstract}
The purpose of this paper is to close one of the last gaps in the classification of finite simple groups containing a standard subgroup. We prove that a simple group containing a standard subgroup of type ${ }^{2} E_{6}(2)$ has to be isomorphic to $F_{2}$, the baby monster.
\end{abstract}

One of the remaining standard form problems is the classification of finite groups containing a standard subgroup of type ${ }^{2} E_{6}(2)$. A quasi-simple group $A$ is said to be a standard subgroup in a group $G$ provided

(i) $N_{G}(A)=N_{G}\left(C_{G}(A)\right)$,

(ii) $\left|C_{G}(A)\right|$ is even,

(iii) $\left|C_{G}(A) \cap C_{G}(A)^{g}\right|$ is odd for all $g \in G-N_{G}(A)$,

(iv) $\left[A, A^{g}\right] \neq 1$ for all $g \in G$.

The purpose of this paper is to handle the case $A / Z(A) \simeq{ }^{2} E_{6}(2)$ and 2||$Z(A) \mid$. Further we may assume $m_{2}\left(C_{G}(A)\right)=1$. Otherwise a result due to $M$. Aschbacher and G. Seitz [2] yields $A \leqslant G$. Furthermore a Sylow 2-subgroup of $C_{G}(A)$ is cyclic. Otherwise the classical involution theorem due to M. Aschbacher [1] yields $A \leqslant G$. The case $A / Z(A) \cong{ }^{2} E_{6}(2)$ and $|Z(A)|$ odd has been treated by G. Seitz in [8]. In this paper we prove

THEOREM. Let $G$ be a finite group, $O(G)=1$, and $A$ a standard subgroup in $G$ such that $A / Z(A) \cong{ }^{2} E_{6}(2)$ and $|Z(A)|$ is even. Then $\left\langle A^{G}\right\rangle=A$ or $\left\langle A^{G}\right\rangle \simeq F_{2}$, the baby monster.

This paper was initiated during the Group Theory conference at the University of California at Santa Cruz in 1979. I wish to thank this institution for its hospitality and the AMS and DFG for their support.

For the properties of ${ }^{2} E_{6}(2)$ used in this paper see [11] and [10]. For the remainder suppose $A \leqslant G$.

\section{Preliminary results.}

(1.1) LEMMA. The group $X={ }^{2} E_{6}(2)$ possesses only one class of elements $\omega$ of order five, $C_{X}(\omega) \cong Z_{5} \times A_{8}$.

Proof. [11, Lemmas (6.10) and (6.2)].

(1.2) Lemma. Let $\nu$ be an element of order 11 in $X={ }^{2} E_{6}(2)$. Then $\left|N_{X}(\langle\nu\rangle)\right|=$ 110 and $\left|C_{X}(\nu)\right|=22$.

Received by the editors November 19, 1979.

1980 Mathematics Subject Classification. Primary 20D05. 
Proof. [11, Lemmas (7.8) and (6.5)].

(1.3) Lemma. Let $z$ be a 2-central involution in $X={ }^{2} E_{6}(2)$. Then

(i) $C_{X}(z)$ is an extension of an extraspecial 2-group $Q$ of width 10 with $P S U_{6}(2)$.

(ii) All involutions of $X$ are conjugated in $X$ to elements of $Q$.

(iii) Let $T$ be a Sylow 2-subgroup of $C_{X}(z)$. Then $J(T /\langle z\rangle)=Q /\langle z\rangle$.

(iv) $C_{T}(z) / Q$ contains exactly one elementary abelian subgroup of order $2^{9}$.

Proof. (i) follows from [10, Lemma 2]; (ii) follows from [10, Lemma 3]. An easy computation using the tables given in [11, pp. 502-505] yields (iii) and (iv).

(1.4) Lemma. Let $X$ be a 2-fold covering group of ${ }^{2} E_{6}(2)$. If $x \in X$ and $x^{2} \in Z(X)$ then $x^{2}=1$.

Proof. [11, p. 503].

(1.5) LEMMA. Let $G$ be a finite group containing an involution $d$ such that $C_{G}(d) / O\left(C_{G}(d)\right)$ is a 2-fold covering of ${ }^{2} E_{6}(2)$. Let $S \in \operatorname{Syl}_{2}\left(C_{G}(d)\right)$. Then $Z(S)$ is elementary abelian of order 4. Let $B$ be a Sylow 2-subgroup of $O_{2^{\prime}, 2}\left(C_{G}(Z(S))\right)$ and $g$ a 2-element normalizing $B$ and acting trivially on $C_{G}(Z(S)) / O_{2^{\prime}, 2}\left(C_{G}(Z(S))\right)$. Then $[g, d]=1$ or

(i) $\langle B, g\rangle=C$ is extraspecial of width 11 and

(ii) $\left|N_{G}(C) / C_{G}(C) C\right|=2^{17} \cdot 3^{6} \cdot 5^{3} \cdot 7 \cdot 11 \cdot 23$.

Proof. $|Z(S)|=4$ follows from Lemmas (1.3) and (1.4). As in the proof of [11, Lemma (4.4)] the existence of a field automorphism of ${ }^{2} E_{6}(2)$ is not used. Application of [11, Lemma (4.4)] proves (i). The same argument is possible for [11, Lemmas (4.6) and (4.7)]. This yields (ii).

\section{Proof of the theorem.}

(2.1) LEMMA. A Sylow 2-subgroup of $C_{G}(A)$ is of order 2.

Proof. Let $T_{1} \in \operatorname{Syl}_{2}(A)$. Then $Z\left(T_{1}\right)=\langle z, d\rangle$ is of order $4,\langle d\rangle \in \operatorname{Syl}_{2}(Z(A))$. Let $Y$ be the preimage of $J\left(T_{1} / Z\left(T_{1}\right)\right)$. Then $Y^{\prime}=\langle z\rangle$, by Lemma (1.3). Let $T \in \operatorname{Syl}_{2}\left(N_{G}(A)\right)$. Then $Z(T)=\langle z, S\rangle, S<C_{G}(A)$. Furthermore $\langle z\rangle$ char $T$. Let $S_{1}=T \cap C_{G}(A)$ and $Y_{1}$ the preimage of $J(T / Z(T))$. Then $Z\left(Y_{1}\right)=\left\langle z, S_{1}\right\rangle$.

Assume $\left|S_{1}\right| \geqslant 4$. Then $T \in \operatorname{Syl}_{2}(G)$. By Lemmas (1.3)(ii) and (1.4) each involution of $A C_{G}(A)$ is conjugate in $A$ to an involution in $Y_{1}$. Let $y \in Y_{1}, d \neq y \sim d$ in G. Then $\left|Y_{1}: C_{Y_{1}}(y)\right|=2$. Let $T_{2}=C_{T}(y) \in \operatorname{Syl}_{2}\left(C_{N_{G}(A)}(y)\right)$ and $T_{3} \subseteq C_{G}(y)$, $\left|T_{3}: T_{2}\right|=2$. As $\Omega_{1}\left(\phi\left(Z\left(C_{Y_{1}}(y)\right)\right)\right)=\langle d\rangle, C_{Y_{1}}(y) \leqslant T_{3}$. Choose $t \in T_{3}-T_{2}$. As $y \sim y z$ and $d \nsim d z$ in $G$ we get $z^{t} \neq z$, otherwise $\langle z, y\rangle=\Omega_{1}\left(Z\left(T_{3}\right)\right) \sim \Omega_{1}(Z(T))$ $=\langle z, d\rangle$. Hence $C_{Y_{1}}(y) \cap C_{Y_{1}}(y)^{t}$ has to be elementary abelian. But this yields that $C_{Y_{1}}(y)^{t} C_{Y_{1}}(y) / C_{Y_{1}}(y)$ is abelian of rank at least 10 . This contradicts Lemma (1.3)(iv) and the structure of $\operatorname{Out}\left({ }^{2} E_{6}(2)\right)$ [9]. Thus $d^{G} \cap A C_{G}(A)=d$. Let $y$ be an involution in $N_{G}(A)-C_{G}(A) A$. Then there is a fours group $V$ contained in $A$ such that $V y \subseteq y^{G}$. Let $g \in G$ with $y^{g}=d$. Then $V^{g} \cap A C_{G}(A) \neq 1$. But this contradicts $d^{G} \cap A C_{G}(A)=d$. Thus $d^{G} \cap N_{G}(A)=d$. Now application of [3] yields the contradiction $A \leqslant G$. 
(2.2) Lemma. $\left|G: N_{G}(A)\right|$ is even.

Proof. Suppose $\left|G: N_{G}(A)\right|$ to be odd. Let $T \in \operatorname{Syl}_{2}\left(N_{G}(A)\right)$. Then $Z(T)=$ $\langle d, z\rangle,\langle d\rangle \in \operatorname{Syl}_{2}(Z(A))$. Let $E$ be the preimage of $J(T / Z(T))$. By Lemma (1.3) we have that $E$ is the direct product of $\langle d\rangle$ with an extraspecial group of width 10 . Furthermore $E^{\prime}=\langle z\rangle$. By Lemma (1.3)(ii) every involution of $A$ is conjugate in $A$ to an involution of $E$. Let $y \neq d$ be an involution in $E$ such that $y \sim d$ in $G$. As $d \nsim z \nsim d z \nsim d, y \sim y z$ in $G$. Further $\left|E: C_{E}(y)\right|=2$. Set $T_{1}=C_{T}(y)$. We may assume $T_{1} \in \operatorname{Syl}_{2}\left(C_{N_{G}(A)}(y)\right)$. Let $T_{2} \leqslant C_{G}(y),\left|T_{2}: T_{1}\right|=2$ and $x \in T_{2}-T_{1}$. Then $z^{x} \neq z$, otherwise $\langle z, y\rangle \sim\langle z, d\rangle$. Hence $C_{E}(y)^{x} \neq C_{E}(y)$. Thus $\left|C_{E}(y)^{x} C_{E}(y) / C_{E}(y)\right| \geqslant 2^{9}$. Now Lemma (1.3)(iv) yields that $C_{E}(y)^{x} C_{E}(y) / C_{E}(y)$ is uniquely determined. But then (see [7, Lemma 1]) $C_{E}\left(C_{E}(y)^{x}\right)$ contains only involutions conjugate to $d, z$ or $d z$ in $A$. This is a contradiction. Hence $d^{G} \cap$ $C_{G}(A) A=d$.

Suppose $y \in N_{G}(A)-A, y \sim d$ in $G$. Then $A$ contains a fours group $V$ such that $V y \leqslant y^{G}$. But this contradicts $d^{G} \cap C_{G}(A) A=d$. Thus $d^{G} \cap N_{G}(A)=d$. Now the application of [3] yields the contradiction $A \leqslant G$.

(2.3) LEMMA. There are involutions in $N_{G}(A)$ acting as field automorphisms on $A / Z(A)$. In particular $\left|N_{G}(A): C_{G}(A) A\right|=2$.

Proof. According to Lemma (2.2) $d \sim d z$ in $G$. Thus there is a 2-element $g$ in $N_{G}(\langle d, z\rangle)$ with $d^{g}=d z$. Suppose $N_{G}(A)=C_{G}(A) A$. By Lemma (1.3)(i) $N_{N_{G}(A)}(\langle d, z\rangle) / O_{2,2^{\prime}}\left(N_{N_{G}(A)}(\langle d, z\rangle)\right) \simeq P S U_{6}(2)$.

Assume

$$
\left[g, N_{N_{G}(A)}(\langle d, z\rangle)\right] \subseteq O_{2,2^{\prime}}\left(N_{N_{G}(A)}(\langle d, z\rangle)\right) .
$$

Set $C=\left\langle O_{2}\left(C_{G}(\langle d, z\rangle)\right), g\right\rangle$. Then Lemma (1.5) yields that $C$ is extraspecial of width 11 and $\left|N_{G}(C) / C_{G}(C) C\right|=2^{17} \cdot 3^{6} \cdot 5^{3} \cdot 7 \cdot 11 \cdot 23$. Let $t$ be a 2-central involution in $C_{G}(\langle d, z\rangle) / O_{2}\left(C_{G}(\langle d, z\rangle)\right)$. Set $\overline{N_{G}(C)}=N_{G}(C) / C_{G}(C) C$ and $\tilde{C}=$ $C /\langle z\rangle$. Now $\bar{X}=O_{2}\left(C_{\overline{N_{G}}(C) \cap N_{G}(A)}(\bar{t})\right)$ is extraspecial of width 4. Furthermore $C_{\overline{N_{G}(C) \cap N_{G}(A)}}(\bar{t}) / \bar{X} \cong U_{4}(2) ;$ Lemma (1.3)(i). The group $\bar{X}$ centralizes in $\tilde{C}$ a subgroup of order $2^{7}$, see [11, Lemma (5.1)]. As $C_{\overline{N_{G}(C) \cap N_{G}(A)}}(\bar{t})$ induces on $(\tilde{Y} /\langle\tilde{d}\rangle)^{\#}$ two orbits of length 27 and 36 , we get that $d$ possesses exactly 28 conjugates under the action of $N_{\overline{N_{G}(C)}}(\bar{X})$. Thus $N_{\overline{N_{G}(C)}}(\bar{X}) / \bar{X}$ is of order $2^{8} \cdot 3^{4} \cdot 5$ - 7. As $U_{4}(2)$ is involved we get that this group has to be simple. Checking the list of groups in [5] we get a contradiction. Thus we have that $\boldsymbol{g}$ induces an outer automorphism on $N_{N_{G}(A)}(\langle d, z\rangle) / O_{2,2^{2}}\left(N_{N_{G}(A)}(\langle d, z\rangle)\right)$. Then by Lemma (1.3)(i) $O_{2}\left(N_{N_{G}(A)}(\langle d, z\rangle)\right) /\langle z\rangle$ is the unique elementary abelian subgroup of order $2^{21}$ in a Sylow 2-subgroup of $N_{G}(\langle d, z\rangle) /\langle z\rangle$. Hence $d^{C_{G}(z)} \cap O_{2}\left(N_{N_{G}(A)}(\langle d, z\rangle)\right)=$ $\{d, d z\}$.

Assume now $O\left(N_{G}(A)\right) \neq 1$. Let $T$ be a Sylow 2-subgroup of $A$ containing $z$. Then $C_{G}(T)=O\left(N_{G}(A)\right)\langle z, d\rangle$. Thus $d \sim d z$ in $N_{G}\left(O\left(N_{G}(A)\right)\right)$. Now by induction we get that $A O\left(N_{G}\left(O\left(N_{G}(A)\right)\right)\right)$ is normal in $N_{G}\left(O\left(N_{G}(A)\right)\right)$. But this is a contradiction. Thus $O\left(N_{G}(A)\right)=1$.

Let $\omega$ be an element of order five in $C_{G}(z) \cap N_{G}(A)$. By Lemma (1.1) we may 
assume $d \sim d z$ in $C_{G}(\omega)$. By Lemmas (1.1) and (1.4) we have $C_{A}(\omega) /\langle\omega\rangle \simeq\langle d\rangle \times$ $A_{8}$. Let $S$ be a Sylow 2-subgroup of $C_{A}(\omega)$. Then $S$ contains an elementary abelian subgroup $B$ of order 32. Because of $d^{C_{G}(z)} \cap O_{2}\left(N_{A}(\langle d, z\rangle)\right)=\{d, d z\}$ we get that $d$ has exactly 10 conjugates under $N_{C_{G}(\omega)}(B)$. As $A_{6}$ contains no subgroup isomorphic to $\Sigma_{3} \times \Sigma_{3}$ we get $O_{3}\left(N_{C_{G}(\omega)}(B) / C_{C_{G}(\omega)}(B)\right)$ is nontrivial. But then we get the contradiction that $d$ is weakly closed in $B$ with respect to $N_{C_{G}(\omega)}(B)$.

Hence we have shown that $\left|N_{G}(A): C_{G}(A) A\right|=2$. Then we may assume that $\left[g, N_{N_{G}(A)}(\langle d, z\rangle)\right] \subseteq O_{2,2^{\prime}}\left(N_{N_{G}(A)}(\langle d, z\rangle)\right)$, as $\operatorname{Out}\left(P S U_{6}(2)\right) \cong \Sigma_{3}$, see [9]. By Lemma (1.2) we may assume that $g$ centralizes an element $\nu$ in $N_{N_{G}(A)}(\langle d, z\rangle)$ with $\nu^{11} \in O\left(C_{G}(A)\right)$. By Lemma (1.2) we get that a Sylow 2-subgroup $S_{1}$ of $N_{G}(\langle\nu\rangle) \cap$ $N_{N_{G}(A)}(\langle d, z\rangle)$ is of order eight. Clearly $S_{1}$ is abelian. We may assume that $g$ normalizes $S_{1}$. Thus $d \notin \phi\left(S_{1}\right)$. Then $S_{1}$ has to be elementary abelian. Thus there is an involution in $N_{G}(A)-C_{G}(A)$ normalizing a subgroup of order 11 in $A$. Now the structure of $\operatorname{Aut}\left({ }^{2} E_{6}(2)\right)$ [9] yields that this involution induces a field automorphism on $A / Z(A)$.

(2.4) LEMMA. We have $\left\langle A^{G}\right\rangle \simeq F_{2}$.

Proof. By Lemma (2.3) and [11] it is enough to show $O\left(N_{G}(A)\right)=1$. Suppose $K=O\left(N_{G}(A)\right) \neq 1$. Let $C=F^{*}\left(C_{A}(\langle d, z\rangle)\right)$. As $d \sim d z$ in $G, d \sim d z$ in $N_{G}(C)$. We have $C_{G}(C)=K\langle d, z\rangle$. Thus $d \sim d z$ in $N_{G}(K)$. As $A<N_{G}(K)$ we get by induction $N_{G}(K) / O\left(N_{G}(K)\right) \cong F_{2}$ and $\left[K,\left\langle A^{N_{G}(K)}\right\rangle\right]=1$. Let $Y$ be a Sylow 2-subgroup of $F^{*}\left(C_{N_{G}(K)}(z)\right)$. Then $Y$ is extraspecial of width 11 . The conjugacy classes of involutions in $N_{N_{G}(K)}(Y)-Y$ are listed in [11, Table VI]. Let $x$ be such an involution. Then it is easy to see that $C_{N_{N_{G}(K)}(Y)}(T)=Z(T) \times K$, for $T \in$ $\operatorname{Syl}_{2}\left(C_{N_{N_{G}(K)}(Y)}(x)\right)$. Thus $d \nsim x$ in $C_{G}(z)$. Thus the weak closure of $d$ in $N_{G}(Y)$ with respect to $C_{G}(z)$ is contained in $Y$. Then $Y$ is strongly closed in $N_{G}(Y)$ with respect to $C_{G}(z)$. Application of [4] yields now $C_{G}(z) \subseteq N_{G}(K)$. Now the structure of centralizers of involutions in $N_{G}(K)$ yields that $N_{G}(K)$ controls $G$-fusion of 2-central involutions in $N_{G}(K)$. But then $G=N_{G}(K)$ by Holt's theorem [6]. This contradicts $O(G)=1$. The lemma is proved.

\section{REFERENCES}

1. M. Aschbacher, A characterization of Chevalley groups over fields of odd characteristic, Ann. of Math. 106 (1977), 353-468.

2. M. Aschbacher and G. Seitz, On groups with a standard component of known type, Osaka J. Math. 13 (1976), 439-482.

3. G. Glauberman, Central elements in core free groups, J. Algebra 4 (1966), 403-421.

4. D. M. Goldschmidt, 2-fusion in finite groups, Ann. of Math. 99 (1974), 70-117.

5. M. Hall, Simple groups of order less than one million, J. Algebra 20 (1972), 98-102.

6. D. Holt, Transitive permutation groups in which an involution central in a Sylow 2-subgroup fixes a unique point, Proc. London Math. Soc. 37 (1978), 165-192.

7. A. Reifart, On finite simple groups with large extraspecial subgroups, I. J. Algebra 53 (1978), 452-470.

8. G. Seitz, Chevalley groups as standard subgroups, I. Illinois J. Math. 23 (1979), 36-57.

9. R. Steinberg, Automorphisms of finite linear groups, Canad. J. Math. 12 (1960), 606-615.

10. G. Stroth, Eine Kennzeichnung der Gruppe ${ }^{2} E_{6}\left(2^{n}\right)$, J. Algebra 35 (1975), 534-547.

11. $\ldots$ A characterization of Fischer's sporadic simple group of the order $2^{41} \cdot 3^{13} \cdot 5^{6} \cdot 7^{2} \cdot 11 \cdot 13$. $17 \cdot 19 \cdot 23 \cdot 31 \cdot 47$, J. Algebra 40 (1976), 499-531.

Freie Universträt Berlin, II. Mathematisches Instrtut, Königin-Luise-Strasse 24-26, D-1000 BerLin 33, Germany 